\title{
Static Bending Analysis of Auxetic Plate by FEM and a New Third-Order Shear Deformation Plate Theory
}

\author{
Pham Hong Cong ${ }^{1, *}$, Pham Minh Phuc ${ }^{2}$, Hoang Thi Thiem³, \\ Duong Tuan Manh ${ }^{4}$, Nguyen Dinh Duc ${ }^{4}$ \\ ${ }^{I}$ Centre for Informatics and Computing (CIC), Vietnam Academy of Science and Technology, \\ 18 Hoang Quoc Viet, Hanoi, Vietnam \\ ${ }^{2}$ Faculty of Basic Sciences, University of Transport and Communications, \\ 03 Cau Giay, Dong Da, Hanoi, Vietnam \\ ${ }^{3}$ VNU University of Sciences, Vietnam National University, Hanoi, Department of Mathematics, \\ Mechanics and Informatics, 334 Nguyen Trai, Hanoi, Vietnam \\ ${ }^{4}$ VNU University of Engineering and Technology, Vietnam National University, Hanoi, \\ Department of Engineering and Technology of Constructions and Transportation, \\ 144 Xuan Thuy, Hanoi, Vietnam \\ Received 16 February 2020 \\ Revised 01 March 2020; Accepted 01 March 2020
}

\begin{abstract}
In this paper, a finite element method (FEM) and a new third-order shear deformation plate theory are proposed to investigate a static bending model of auxetic plates with negative Poisson's ratio. The three - layer sandwich plate is consisted of auxetic honeycombs core layer with negative Poisson's ratio integrated, isotropic homogeneous materials at the top and bottom of surfaces. A displacement-based finite element formulation associated with a novel third-order shear deformation plate theory without any requirement of shear correction factors is thus developed. The results show the effects of geometrical parameters, boundary conditions, uniform transverse pressure on the static bending of auxetic plates with negative Poisson's ratio. Numerical examples are solved, then compared with the published literatures to validate the feasibility and accuracy of proposed analysis method.
\end{abstract}

Keywords: Static bending; new third-order shear deformation plate theory; auxetic material.

\footnotetext{
* Corresponding author.

Email address: phcong@ cic.vast.vn

https://doi.org/10.25073/2588-1140/vnunst.5000
} 


\section{Introduction}

Auxetic materials are fascinating materials which, when placed under tension in one direction, become thicker in one or more perpendicular directions (Figure 1). In other words, an auxetic material possesses a negative value of Poisson's ratio (Evans et al. [1]).

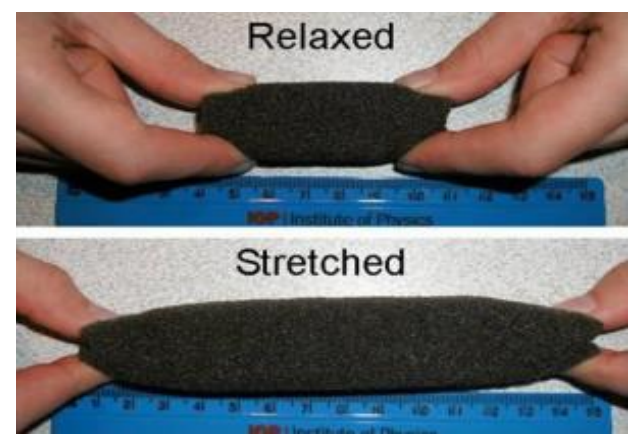

Figure 1. Auxetic material [2].

Recently, numerous investigations on auxetic materials have been conducted by researchers in all over the world. The mechanical behaviors such as static bending, bucking load, dynamic response and vibration are studied a lot. Shariyat and Alipour [3] investigated bending and stress analysis of variable thickness FGM auxetic conical/cylindrical shells with general tractions (using first-order shear-deformation theory and ABAQUS finite element analysis code). The only published paper on stress analysis of the auxetic structures was due to Alipour and Shariyat [4] who developed analytical zigzag solutions with 3D elasticity corrections for bending and stress analysis of circular/annular composite sandwich plates with auxetic cores. Hou et al. [5] studied the bending and failure behaviour of polymorphic honeycomb topologies consisting of gradient variations of the horizontal rib length and cell internal across the surface of the cellular structures. The novel cores were used to manufacture sandwich beams subjected to three-point bending tests. Full-scale nonlinear Finite Element models were also developed to simulate the flexural and failure behaviour of the sandwich structures.

Auxetic plate and shell structures under blast load are mainly studied in nonlinear dynamic response and vibration problems. The calculus, semi-calculus, and numerical methods are proposed. There are a variety of studies applied analytical methods including the authors Duc and Cong [6-10]. In [6-10], the analytical Reddy's (first or third) order shear deformation theory with the geometrical nonlinear in von Karman and Airy stress functions, Galerkin and the fourth-order Runge-Kutta methods were proposed to consider cell of honeycomb core layer (with NPR). Specifically, the nonlinear dynamic response of auxetic plate was conducted in [6], cylinder auxetic shell (within and without stiffeners) was illustrated in $[7,10]$ and double curved shallow auxetic shells (without stiffeners) were mentioned in $[8,9]$.

From above literature review, in [3-5] the authors conducted bending and stress analysis auxetic structures using first-order shear strain theory and finite element method while in [610], an analytical method and (first or higher) order shear deformation theory were proposed to study dynamic response and vibration of auxetic plate and shell structures.

To the author's best knowledge, a new thirdorder shear deformation plate theory has not been used in any published literature yet and it is also the main motivation of this research work. It introduces static bending analysis of auxetic plates with negative Poisson's ratio using FEM and a new third-order shear deformation plate theory. The results show the effects of geometrical parameters, boundary conditions, uniform transverse pressure on the static bending of auxetic plates with negative Poisson's ratio.

\section{Sandwich plate with auxetic core}

Considering a sandwich plate with auxetic core which has three layers in which the top and bottom outer skins are isotropic aluminum materials; the central layer has honeycomb structure using the same aluminum material (Figure 2a). The bottom outer skin thickness is $h_{1}$, internal honeycomb core material thickness is $h_{2}$ and top outer skin thickness is $h_{3}$, and the total thickness of the sandwich plate is $h=h_{1}+h_{2}+h_{3}$, as shown in Figure 2b. 


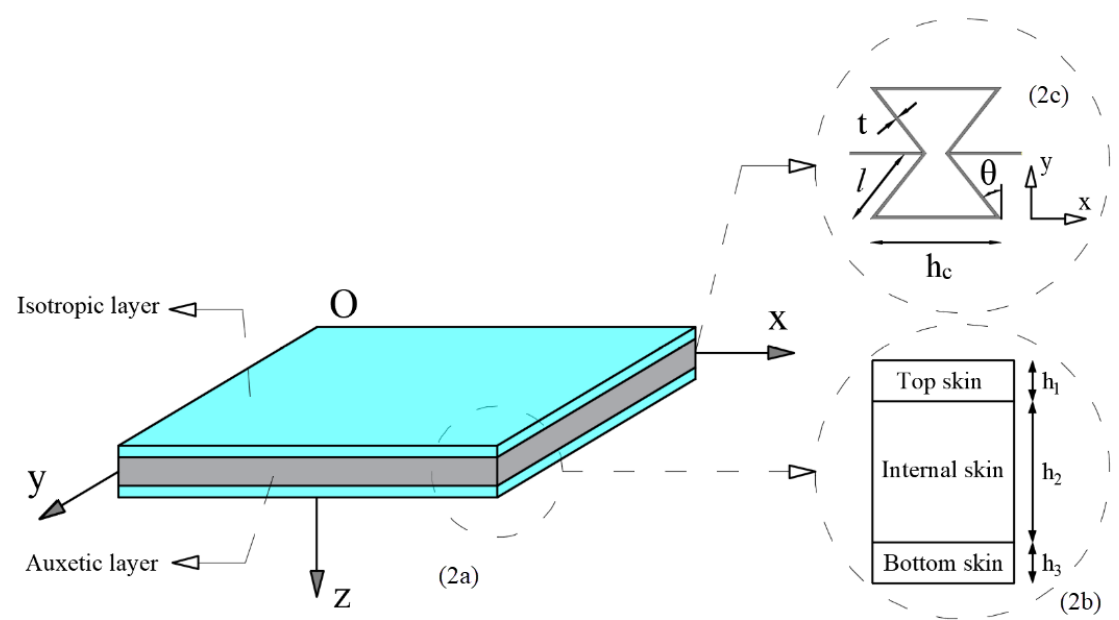

Figure 2. Model of sandwich plate with auxetic core.

The plate with the auxetic honeycomb core with negative Poisson's ratio is introduced in this paper. Unit cells of core material discussed in the paper are shown in Figure 2c where $l$ is the length of the inclined cell rib, $h_{c}$ is the length of the vertical cell rib, $\theta$ is the inclined angle, $\alpha$ and $\beta$ define the relative cell wall length and the wall's slenderness ratio, respectively, which are important parameters in honeycomb property.

Formulas in reference [11] are adopted for calculation of honeycomb core material property.

$$
\begin{aligned}
& E_{1}^{(2)}=E \frac{n_{3}^{3}\left(\eta_{1}-\sin \theta\right)}{\cos \theta^{3}\left[1+\left(\tan ^{2} \theta+\eta_{1} \sec ^{2} \theta\right) \eta_{3}^{2}\right]} \\
& E_{2}^{(2)}=E \frac{n_{3}^{3}}{\cos \theta\left(\eta_{1}-\sin \theta\right)\left(\tan ^{2} \theta+\eta_{3}^{2}\right)} \\
& G_{12}^{(2)}=E \frac{\eta_{3}^{3}}{\eta_{1}\left(1+2 \eta_{1}\right) \cos \theta} \\
& G_{23}^{(c)}=G \frac{\eta_{3} \cos \theta}{\eta_{1}-\sin \theta} \\
& G_{13}^{(2)}=G \frac{\eta_{3}}{2 \cos \theta}\left[\frac{\eta_{1}-\sin \theta}{1+2 \eta_{1}}+\frac{\eta_{1}+2 \sin { }^{2} \theta}{2\left(\eta_{1}-\sin \theta\right)}\right]
\end{aligned}
$$

$$
\begin{aligned}
& v_{12}^{(2)}=-\frac{\sin \theta\left(1-n_{3}^{2}\right)\left(\eta_{1}-\sin \theta\right)}{\cos ^{2} \theta\left[1+\left(\tan ^{2} \theta+\sec ^{2} \theta \eta_{1}\right) \eta_{3}^{2}\right]} \\
& v_{21}^{(2)}=-\frac{\sin \theta\left(1-\eta_{3}^{2}\right)}{\left(\tan ^{2} \theta+\eta_{3}^{2}\right)\left(\eta_{1}-\sin \theta\right)} \\
& \rho^{(2)}=\rho \frac{\eta_{3}\left(\eta_{1}+2\right)}{2 \cos \theta\left(\eta_{1}-\sin \theta\right)} \\
& \left(\eta_{1}=h / l, \eta_{3}=t / l\right) .
\end{aligned}
$$

where symbol "(2)" represents core material, $E, G$ and $\rho$ are Young's moduli, shear moduli and mass density of the origin material.

\section{New simple third-order shear deformation theory of plates}

A finite element formulation based on a new third-order shear deformation plate theory, which is originally proposed by Shi in [12], for static bending analysis of auxetic plates is derived in this section. This new plate theory, in which the kinematic of displacements is derived from an elasticity formulation rather than the hypothesis of displacements, has shown more accurate than other higher-order shear deformation plate theories. The displacements, 
$u, v$ and $w$ at any point of the plate are given by [12].

$$
\begin{aligned}
& u=u_{0}+\frac{5}{4}\left(z-\frac{4}{3 h^{2}} z^{3}\right) \phi_{x} \\
& +\left(\frac{1}{4} z-\frac{5}{3 h^{2}} z^{3}\right) w_{0, x} \\
& v=v_{0}+\frac{5}{4}\left(z-\frac{4}{3 h^{2}} z^{3}\right) \phi_{y} \\
& +\left(\frac{1}{4} z-\frac{5}{3 h^{2}} z^{3}\right) w_{0, \mathrm{y}} \\
& w=w_{0}
\end{aligned}
$$

where $u_{0}, v_{0}$, and $w_{0}$ are respectively the displacements in the $x, y$ and $Z$ directions of a point on the mid-plane of a plate, while $\phi_{x}$ and $\phi_{y}$ denote the transverse rotations of a midsurface normal around the $x$ and $y$ axes, respectively.

Under small strain assumptions, the straindisplacement relations can be expressed as follows:

$$
\begin{aligned}
& \left\{\begin{array}{l}
\varepsilon_{x} \\
\varepsilon_{y} \\
\varepsilon_{x y} \\
\gamma_{y z} \\
\gamma_{x z}
\end{array}\right\}=\left\{\begin{array}{l}
\varepsilon^{(0)} \\
\gamma^{(0)}
\end{array}\right\}+z\left\{\begin{array}{l}
\varepsilon^{(1)} \\
0
\end{array}\right\}+z^{2}\left\{\begin{array}{l}
0 \\
\gamma^{(2)}
\end{array}\right\} \\
& +z^{3}\left\{\begin{array}{l}
\varepsilon^{(3)} \\
0
\end{array}\right\}
\end{aligned}
$$

in which

$$
\varepsilon^{(0)}=\left\{\begin{array}{l}
\frac{\partial u_{0}}{\partial x} \\
\frac{\partial v_{0}}{\partial y} \\
\frac{\partial v_{0}}{\partial x}+\frac{\partial u_{0}}{\partial y}
\end{array}\right\}
$$

$$
\begin{gathered}
\varepsilon^{(1)}=\frac{1}{4}\left\{\begin{array}{l}
5 \frac{\partial \phi_{x}}{\partial x}+\frac{\partial^{2} w}{\partial x^{2}} \\
5 \frac{\partial \phi_{y}}{\partial y}+\frac{\partial^{2} w}{\partial y^{2}} \\
5 \frac{\partial \phi_{x}}{\partial y}+2 \frac{\partial^{2} w}{\partial x \partial y}+5 \frac{\partial \phi_{y}}{\partial x}
\end{array}\right\} \\
\varepsilon^{(3)}=\frac{-5}{3 h^{2}}\left\{\begin{array}{l}
\frac{\partial \phi_{x}}{\partial x}+\frac{\partial^{2} w}{\partial x^{2}}+\frac{\partial^{2} w}{\partial y^{2}} \\
\frac{\partial \phi_{x}}{\partial y}+2 \frac{\partial^{2} w}{\partial x \partial y}+\frac{\partial \phi_{y}}{\partial x}
\end{array}\right\} \\
\gamma^{(0)}=\frac{5}{4}\left\{\begin{array}{l}
\frac{\partial w}{\partial y}+\phi_{y} \\
\frac{\partial w}{\partial x}+\phi_{x}
\end{array}\right\} \\
\gamma^{(2)}=\frac{-5}{h^{2}}\left\{\begin{array}{l}
\frac{\partial w}{\partial y}+\phi_{y} \\
\frac{\partial w}{\partial x}+\phi_{x}
\end{array}\right\}
\end{gathered}
$$

Based on Hooke's law, the vectors of normal and shear stresses read

$$
\begin{aligned}
& \sigma^{(k)}=D_{m}^{(k)}\left(\varepsilon^{(0)}+z \varepsilon^{(1)}+z^{3} \varepsilon^{(3)}\right) \\
& \tau^{(k)}=D_{s}^{(k)}\left(\gamma^{(0)}+z^{2} \gamma^{(2)}\right)
\end{aligned}
$$

with

$$
\begin{aligned}
& \sigma^{(k)}=\left[\begin{array}{lll}
\sigma_{x}^{(k)} & \sigma_{y}^{(k)} & \sigma_{x y}^{(k)}
\end{array}\right]^{T} \\
& \tau^{(k)}=\left[\begin{array}{ll}
\tau_{y z}^{(k)} & \tau_{x z}^{(k)}
\end{array}\right]^{T} \\
& D_{m}^{(k)}=\left[\begin{array}{ccc}
Q_{11}^{(k)} & Q_{12}^{(k)} & 0 \\
Q_{12}^{(k)} & Q_{22}^{(k)} & 0 \\
0 & 0 & Q_{66}^{(k)}
\end{array}\right]
\end{aligned}
$$




$$
D_{s}=\left[\begin{array}{cc}
Q_{55}^{(k)} & 0 \\
0 & Q_{44}^{(k)}
\end{array}\right]
$$

$Q_{11}^{(2)}=\frac{E_{1}^{(2)}}{1-v_{12}^{(2)} v_{21}^{(2)}}, Q_{12}^{(2)}=\frac{v_{12}^{(2)} E_{2}^{(2)}}{1-v_{12}^{(2)} v_{21}^{(2)}}$,

$Q_{22}^{(2)}=\frac{E_{2}^{(2)}}{1-v_{12}^{(2)} v_{21}^{(2)}}, Q_{66}^{(2)}=G_{12}^{(2)}$,

$Q_{44}^{(2)}=G_{23}^{(2)}, Q_{55}^{(2)}=G_{13}^{(2)}$,

$Q_{55}^{(2)}=G_{13}^{(2)}, Q_{11}^{(1)}=Q_{22}^{(1)}=\frac{E}{1-v^{2}}$,

$Q_{12}^{(1)}=\frac{v E}{1-v^{2}}, Q_{66}^{(1)}=Q_{44}^{(1)}=Q_{55}^{(1)}=\frac{E}{2(1+v)}$.

The normal forces, bending moments, higher-order moments and shear force can then be computed through the following relations

$$
\begin{aligned}
& \bar{N}=\left\{\begin{array}{lll}
N_{x} & N_{y} & N_{x y}
\end{array}\right\}^{T}= \\
& \sum_{k=1}^{3} \int_{\bar{h}_{k}}^{\bar{h}_{k+1}}\left\{\begin{array}{lll}
\sigma_{x} & \sigma_{y} & \sigma_{x y}
\end{array}\right\}^{T} d z
\end{aligned}
$$

$$
=\sum_{k=1}^{3} \int_{\bar{h}_{k}}^{\bar{h}_{k+1}} D_{m}^{(k)}\left(\varepsilon^{(0)}+z \varepsilon^{(1)}+z^{3} \varepsilon^{(3)}\right) d z
$$$$
\bar{M}=\left\{\begin{array}{lll}
M_{x} & M_{y} & M_{x y}
\end{array}\right\}^{T}
$$$$
=\sum_{k=1}^{3} \int_{\bar{h}_{k}}^{\bar{h}_{k+1}}\left\{\begin{array}{lll}
\sigma_{x} & \sigma_{y} & \sigma_{x y}
\end{array}\right\}^{T} z d z
$$

$$
\begin{aligned}
& =\sum_{k=1}^{3} \int_{h_{k}}^{\bar{h}_{k+1}} D_{m}^{(k)}\left(\varepsilon^{(0)}+z \varepsilon^{(1)}+z^{3} \varepsilon^{(3)}\right) z d z \\
& \bar{P}=\left\{\begin{array}{lll}
P_{x} & P_{y} & P_{x y}
\end{array}\right\}^{T}
\end{aligned}
$$

$$
\begin{aligned}
& =\sum_{k=1}^{3} \int_{\bar{h}_{k}}^{\bar{h}_{k+1}}\left\{\begin{array}{lll}
\sigma_{x} & \sigma_{y} & \sigma_{x y}
\end{array}\right\}^{T} z^{3} d z \\
& =\sum_{k=1}^{3} \int_{\bar{h}_{k}}^{\bar{h}_{k+1}} D_{m}^{(k)}\left(\varepsilon^{(0)}+z \varepsilon^{(1)}+z^{3} \varepsilon^{(3)}\right) z^{3} d z \\
& \bar{Q}=\left\{Q_{y} Q_{x}\right\}^{T} \\
& =\sum_{k=1}^{3} \int_{\bar{h}_{k}}^{\bar{h}_{k+1}}\left\{\tau_{y z} \tau_{x z}\right\}^{T} d z \\
& =\sum_{k=1}^{3} \int_{\bar{h}_{k+1}}^{\bar{h}_{k}} D_{s}^{(k)}\left(\gamma^{(0)}+z^{2} \gamma^{(2)}\right) d z \\
& \bar{R}=\left\{R_{y} R_{x}\right\}^{T} \\
& =\sum_{k=1}^{3} \int_{\bar{h}_{k+1}}^{\sum_{h_{k}}}\left\{\tau_{y z} \tau_{x z}\right\}^{T} z^{2} d z \\
& =\sum_{k=1}^{3} \int_{\bar{h}_{k+1}}^{\int_{h}} D_{s}^{(k)}\left(\gamma^{(0)}+z^{2} \gamma^{(2)}\right) z^{2} d z
\end{aligned}
$$

Eqs. (7) can be rewritten in matrix form

$$
\left\{\begin{array}{l}
\bar{N} \\
\bar{M} \\
\bar{P} \\
\bar{Q} \\
\bar{R}
\end{array}\right\}=\left[\begin{array}{ccccc}
A & B & E & 0 & 0 \\
B & D & F & 0 & 0 \\
E & F & H & 0 & 0 \\
0 & 0 & 0 & \bar{A} & \bar{B} \\
0 & 0 & 0 & \bar{B} & \bar{D}
\end{array}\right]\left\{\begin{array}{c}
\varepsilon^{(0)} \\
\varepsilon^{(1)} \\
\varepsilon^{(3)} \\
\gamma^{(0)} \\
\gamma^{(2)}
\end{array}\right\}
$$

where

$$
\begin{aligned}
& (A, B, D, E, F, H)=\int_{-h / 2}^{h / 2}\left(1, z, z^{2}, z^{3}, z^{4}, z^{6}\right) D_{m} d z \\
& (\bar{A}, \bar{B}, \bar{D})=\int_{-h / 2}^{h / 2}\left(1, z^{2}, z^{4}\right) D_{s} d z
\end{aligned}
$$


The deformation energy of auxetic plate has the form:

$$
\begin{aligned}
& U(d)=\frac{1}{2} \int_{\Omega}\left(\begin{array}{l}
\varepsilon^{(0) T} A \varepsilon^{(0)}+\varepsilon^{(0) T} B \varepsilon^{(1)} \\
+\varepsilon^{(0) T} E \varepsilon^{(3)}
\end{array}\right. \\
& +\varepsilon^{(1) T} B \varepsilon^{(0)}+\varepsilon^{(1) T} D \varepsilon^{(1)}+\varepsilon^{(1) T} F \varepsilon^{(3)} \\
& +\varepsilon^{(3) T} E \varepsilon^{(0)}+\varepsilon^{(3) T} F \varepsilon^{(1)}+\varepsilon^{(3) T} H \varepsilon^{(3)} \\
& \left.\begin{array}{l}
+\gamma^{(0) T} \bar{A} \gamma^{(0)}+\gamma^{(0) T} \bar{B} \gamma^{(2)} \\
+\gamma^{(2) T} \bar{B} \gamma^{(0)}+\gamma^{(2) T} \bar{D} \gamma^{(2)}
\end{array}\right) d \Omega
\end{aligned}
$$

For static bending analysis, the bending solutions can be obtained by solving the following equation:

$$
K d=F
$$

where $K$ is the stiffness matrix, $F$ is force vector while $d$ stands for the unknown vector.

\section{Numerical results and discussion}

Both the simply supported and fully clamped boundary conditions are investigated. For the simply supported boundary conditions (SSSS) [13]:

$$
\begin{aligned}
& v_{0}=w=\phi_{y}=0, \text { at } x=0, a \\
& u_{0}=w=\phi_{x}=0, \text { at } y=0, b
\end{aligned}
$$

and the fully clamped edges (CCCC) [13]:

$$
\begin{aligned}
& u_{0}=v_{0}=w=\phi_{x}=\phi_{y}=0 \\
& \partial w / \partial x=\partial w / \partial y=0
\end{aligned}
$$

at $x=0, a$ and $y=0, b$

In this section, the parameters are selected as: $a=1 m, b=1 m, h=a / 20, h_{2}=3 / 5 h$,

$$
\begin{aligned}
& h_{1}=h / 5, E=69 G P a, v=0.33, \theta=45^{\circ}, \\
& \eta_{1}=1.8, \eta_{3}=0.0138571 .
\end{aligned}
$$

4.1. Comparison with the results of the isotropic uniformity calculation

We consider a simply-supported and clamped square plate (side $a=1$ ) under uniform transverse pressure $(F=1)$, and thickness $h$. The modulus of elasticity is taken $E=10,9201$ and the Poisson's ratio is taken as $v=0.3$. The nondimensional transverse displacement is set as

$$
\bar{w}=w \frac{D}{P l^{4}}
$$

where the bending stiffness $\mathrm{D}$ is taken as

$$
D=\frac{E h^{3}}{12\left(1-v^{2}\right)}
$$

The results compared with those of Ferreira [14] are shown in Table 1. In Ref. [14], the author used the theory of Mindlin plate considering for the Q4 element. From table 1, it can be seen a very small difference between 2 studies shows the reliability of the calculation program.

Table 1. Comparison of non-dimensional transverse displacement of a square plate, under uniform pressuresimply-support (SSSS) and clamped (CCCC) boundary conditions

\begin{tabular}{clcccc}
\hline \multirow{2}{*}{$a / h$} & \multirow{2}{*}{ Mesh } & \multicolumn{2}{c}{ SSSS } & \multicolumn{2}{c}{ CCCC } \\
\cline { 3 - 6 } & & Ref. [14] & Present & Ref. [14] & Present \\
\hline \multirow{4}{*}{10} & $10 \times 6$ & 0.004245 & 0.004429 & 0.001486 & 0.001672 \\
& $20 \times 20$ & 0.004263 & 0.004429 & 0.001498 & 0.001673 \\
& $30 \times 30$ & 0.004270 & 0.004428 & 0.001503 & 0.001673 \\
& $6 \times 6$ & 0.004271 & 0.004428 & 0.001503 & 0.001673 \\
10,000 & $10 \times 10$ & 0.004024 & 0.003944 & 0.001239 & 0.001101 \\
& $20 \times 20$ & 0.004049 & 0.004022 & 0.001255 & 0.001208 \\
& $30 \times 30$ & 0.004059 & 0.004055 & 0.001262 & 0.001252 \\
\end{tabular}




\subsection{Static bending analysis of auxetic plate}

The $20 \times 20$ Q4 mesh is used to mesure static bending analysis of auxetic plate and $\bar{w}$ is the deflection at position $x=0.5 m, y=0.5 \mathrm{~m}$.

To study the effect of the geometric parameters of the plate on the static bending of the auxetic sheet with a negative Poisson's ratio, $b / a=0.5,1,2.0$ and $b / a=0.5,1,2.0$ are chosen. There are 9 different cases of auxetic plate structures considering 2 types of boundary conditions: SSSS and CCCC. The results are illustrated in Table 2. Obviously, with different boundary conditions and the same value of $b / a$ the value of deflections $(\bar{w})$ decreases as the ratio $h / a$ increases (thicker plates) and vice versa. Whereas, in the case the same value of $h / a$, deflections' value $(\bar{w})$ increase when increasing $b / a$ and vice versa.

Table 2. Effect of the ratio $(b / a)$ and on the deflections $(w)$ of the auxetic plate $\left(v_{21}=-0.646756\right)$

\begin{tabular}{cccc}
\hline \multirow{2}{*}{$b / \mathrm{a}$} & $h / a$ & \multicolumn{2}{c}{ Boundary condition } \\
\cline { 3 - 4 } & 0.01 & 0.000136074 & CCCC \\
\hline \multirow{2}{*}{0.5} & 0.05 & $1.72466 \mathrm{e}-006$ & $3.65492 \mathrm{e}-005$ \\
& 0.10 & $4.19861 \mathrm{e}-007$ & $8.6496 \mathrm{e}-007$ \\
& 0.01 & 0.000844483 & $3.11035 \mathrm{e}-007$ \\
1.0 & 0.05 & $8.60749 \mathrm{e}-006$ & 0.000268721 \\
& 0.10 & $1.63836 \mathrm{e}-006$ & $3.75837 \mathrm{e}-006$ \\
& 0.01 & 0.00210822 & $1.01027 \mathrm{e}-006$ \\
2.0 & 0.05 & $2.01738 \mathrm{e}-005$ & 0.000537942 \\
& 0.10 & $3.44499 \mathrm{e}-006$ & $6.88992 \mathrm{e}-006$ \\
& &
\end{tabular}
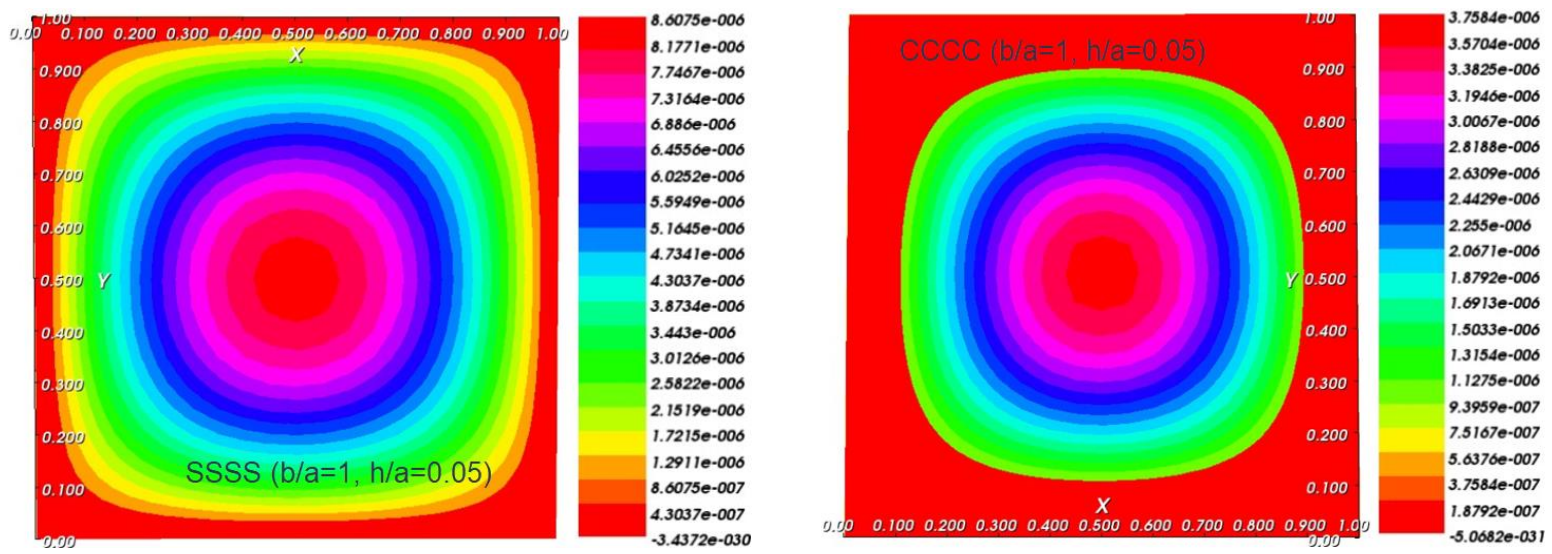

Figure 3. Deformed shape for simply-supported and clamped auxetic plates and $b / a=1, h / a=0.05$ and $v_{21}=-0.646756$. 
Table 3. Calculation values of the deflections $(\bar{w})$ of the auxetic plate with negative Poisson's ratio for different ratios $l / h(F=1000 P a, a / h=20, a=b)$

\begin{tabular}{cccc}
\hline \multirow{2}{*}{$l / h$} & \multirow{2}{*}{$v_{21}^{c}$} & \multicolumn{2}{c}{ Boundary condition } \\
\cline { 3 - 4 } & & SSSS & CCCC \\
\hline 0.2 & -0.164652 & $8.67574 \mathrm{e}-006$ & $3.82713 \mathrm{e}-006$ \\
0.4 & -0.394243 & $8.64811 \mathrm{e}-006$ & $3.7976 \mathrm{e}-006$ \\
0.6 & -0.736624 & $8.59205 \mathrm{e}-006$ & $3.74379 \mathrm{e}-006$ \\
0.8 & -1.30198 & $8.49303 \mathrm{e}-006$ & $3.65204 \mathrm{e}-006$ \\
1 & -2.41329 & $8.31421 \mathrm{e}-006$ & $3.48954 \mathrm{e}-006$ \\
\hline
\end{tabular}

The analysis of the effect of $l / h$ on the deflections $(\bar{w})$ of the auxetic plate consider different values of $l / h=(0.2,0.4,0.6,0.8,1)$. From
Table 3, the increasing in $l / h$ leads to decrease in deflections $(\bar{w})$.
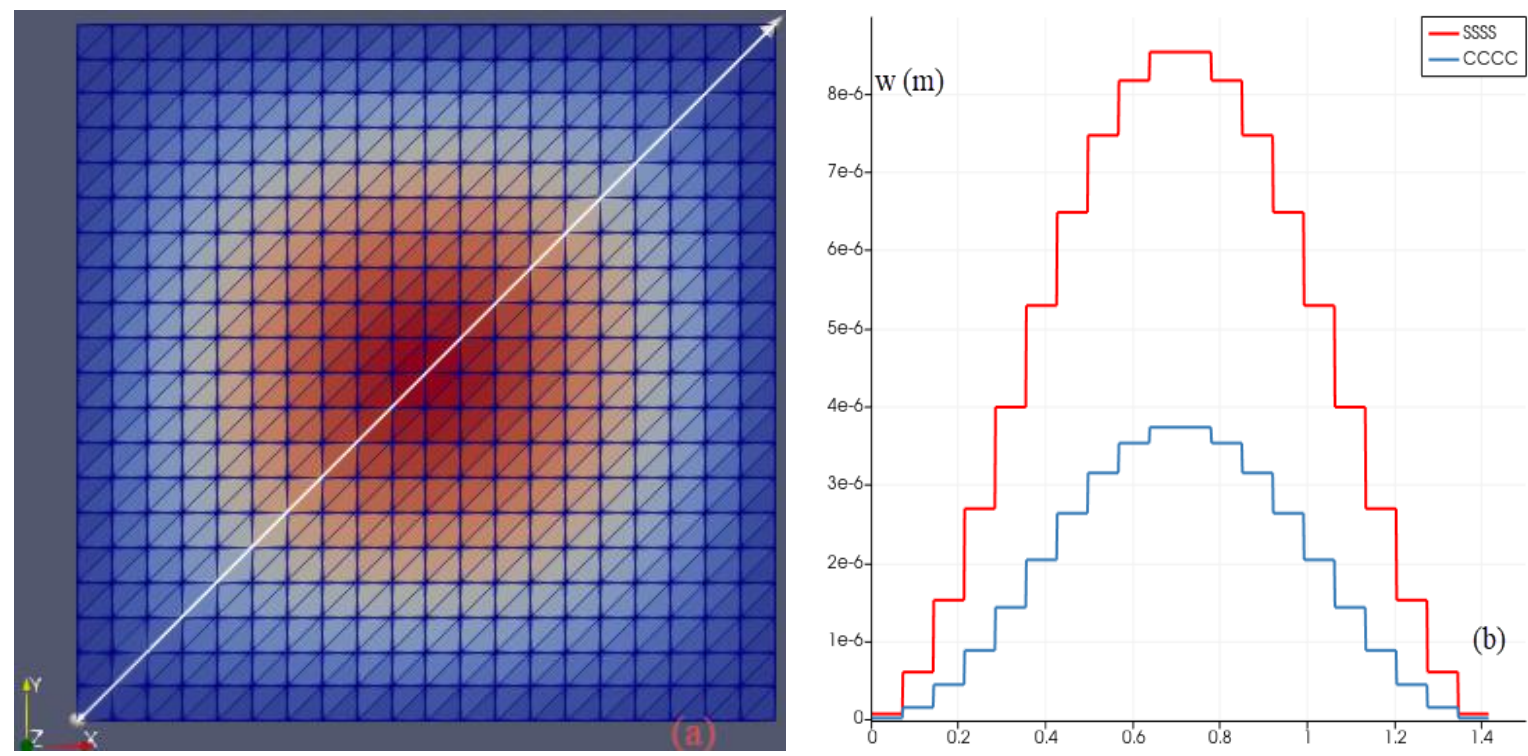

Figure 4 . The deflections $(w)$ of auxetic plates.

Figure $4 \mathrm{~b}$ shows deflections $(w)$ of the nodes in the diagonal direction of the plate as shown in Figure 4a. Figure 4 also illustates that deflections have maximum values at the center of the plate and in the SSSS boundary condition, deflections are larger than those in the CCCC boundary condition. 


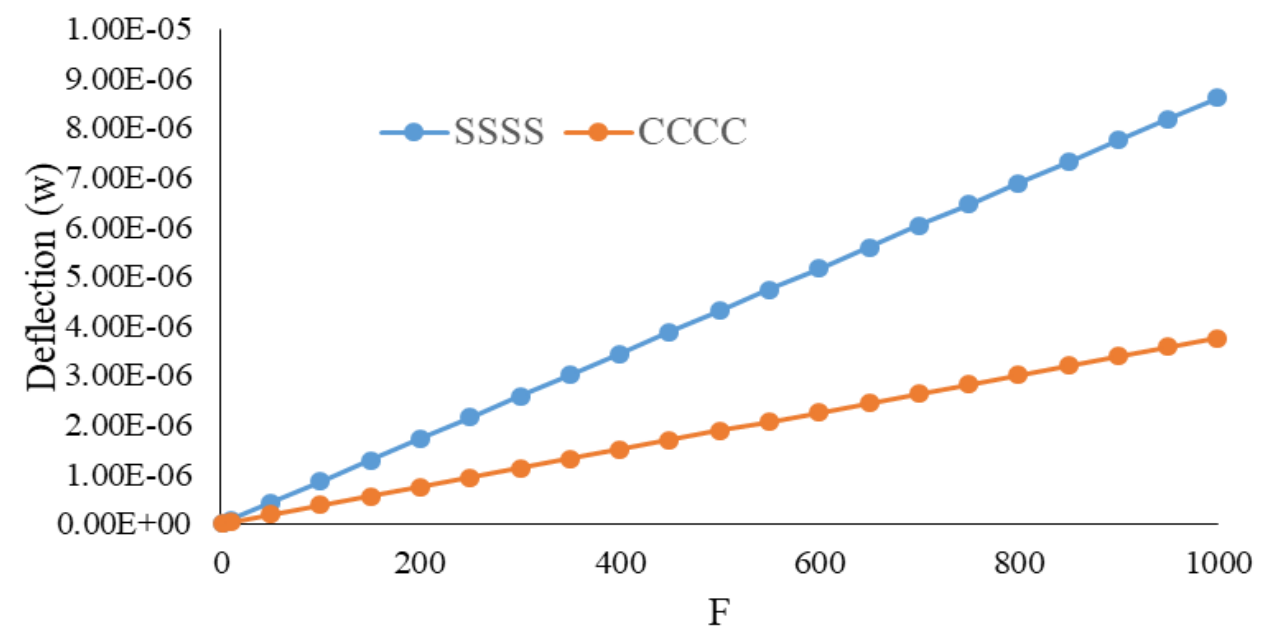

Figure 5. Effect of uniform transverse pressure $F(P a)$ on the deflections $(\bar{w})$ of the auxetic plate

$$
\left(v_{21}=-0.646756\right)
$$

The effect of uniform transverse pressure on the deflections $(\bar{w})$ of the auxetic plate $\left(v_{21}=-0.646756\right)$ is presented in Figure 5. It

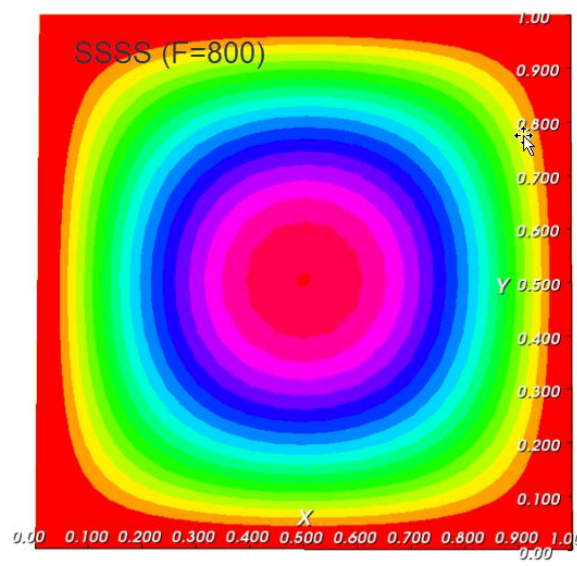

can be seen that increasing the value of uniform transverse pressure makes the value of deflections $(\bar{w})$ and deformed shapes also increase (shown in Figure 6).

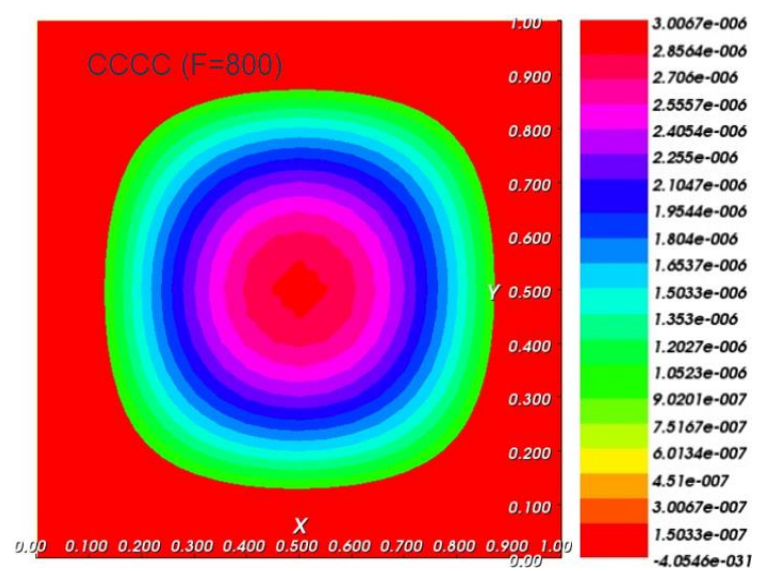

Figure 6. Deformed shape for simply-supported and clamped auxetic plates with value of uniform transverse pressure $F=800 P a$ and $v_{21}=-0.646756$. 


\section{Conclusion}

The paper successfully applied finite element method and a new third-order shear deformation plate theory to study static bending of auxetic plate. The calculation results are compared with other published paper validating the reliability of the calculation program. Then, effect of parameters on static bending of auxetic plates are examined in this paper.

\section{Acknowledgments}

This research is funded by Vietnam National Foundation for Science and Technology Development (NAFOSTED) under grant number 107.02-2019.04.

\section{References}

[1] K.E. Evans, M. Nkansah, I.J. Hutchison, S.C. Rogers, Molecular Network Design, Nature 353 (1991) 124-125. doi:10.1038/353124a0.

[2] S. Mohammad, R. Naveen, A. Kim, A. Andrew, Auxetic materials for sports applications, Procedia Engineering 72 (2014) 453-458. https://doi.org/10. 1016/j.proeng.2014.06.079.

[3] M. Shariyat, M.M. Alipour, Analytical Bending and Stress Analysis of Variable Thickness FGM Auxetic Conical/Cylindrical Shells with General Tractions, Latin American Journal of Solids and Structures 14 (2017) 805-843. http://dx.doi.org/10. 1590/1679-78253413.

[4] M.M. Alipour, M. Shariyat, Analytical zigzag formulation with 3D elasticity corrections for bending and stress analysis of circular/annular composite sandwich plates with auxetic cores, Composite Structures 132 (2015) 175-197. https:// doi.org/10.1016/j.compstruct.2015.05.003.

[5] Y. Hou, Y.H. Tai, C. Lira, F. Scarpa, J.R. Yates, B. $\mathrm{Gu}$, The Bending and Failure of Sandwich Structures with Auxetic Gradient Cellular Cores, Composites Part A: Applied Science and Manufacturing 49(2013) 119-131. https://doi.org/ 10.1016/j.compositesa.2013.02.007.
[6] N.D. Duc, P.H. Cong, Nonlinear dynamic response and vibration of sandwich composite plates with negative Poisson's ratio in auxetic honeycombs, Journal of Sandwich Structures and Materials 20(6) (2018) 692-717. https://doi.org/10.1177/10996362 16674729.

[7] P.H. Cong, P.T. Long, N.V. Nhat, N.D. Duc, Geometrically nonlinear dynamic response of eccentrically stiffened circular cylindrical shells with negative Poisson's ratio in auxetic honeycombs core layer, International journal of Mechanical Sciences 152 (2019) 443-453. https:// doi.org/10.1016/j.ijmecsci.2018.12.052.

[8] P.H. Cong, N.D. Khanh, N.D. Khoa, N.D. Duc, New approach to investigate nonlinear dynamic response of sandwich auxetic double curves shallow shells using TSDT, Compos. Struct. 185 (2018) 455-465. https://doi.org/10.1016/j. compstruct.2017.11.047.

[9] N.D. Duc, K.S. Eock, P.H. Cong, N.T. Anh, N.D. Khoa, Dynamic response and vibration of composite double curved shallow shells with negative Poisson's ratio in auxetic honeycombs core layer on elastic foundations subjected to blast and damping loads, International Journal of Mechanical of Sciences 133 (2017) 504-512. https: //doi.org/10.1016/j.ijmecsci.2017.09.009.

[10] N.D. Duc, K.S. Eock, N.D. Tuan, P. Tran, N.D. Khoa, New approach to study nonlinear dynamic response and vibration of sandwich composite cylindrical panels with auxetic honeycomb core layer, Aerosp. Sci. Technol. 70 (2017) 396-404. https://doi.org/10.1016/j.ast.2017.08.023.

[11] K. Di, X.B. Mao, Free lexural vibration of honeycomb sandwich plate with negative Poisson's ratio simple supported on opposite edges, Acta Materiae Compositae Sinica 33 (2016) 910-920. https://doi.org/10.13801/j.cnki.fhclxb.20151010.001

[12] G. Shi, A new simple third-order shear deformation theory of plates. Int. J. Solids Struct. 44 (2007) 4399-4417. https://doi.org/10.1016/j.ijsolstr.2006. 11.031.

[13] H.T. Hu, B.H. Lin, Buckling optimization of symmetrically laminated plates with various geometries and end conditions, Composite Science and Technology 55 (1995) 277-285. https://doi. org/10.1016/0266-3538(95)00105-0.

[14] A.J.M Ferreira, Matlab coddes for finite element analysis. Solids and Structures. Solid Mechanics and ít applications, Springer, pages: 165-166. 